\title{
Contribution of laboratories in the WHO Eastern Mediterranean Region to the selection of candidate seasonal influenza vaccine, 2010-2015
}

\author{
H. Asghar', H.M. Browne ${ }^{2}$, J. McCauley ${ }^{3}$, M. Malik ${ }^{7}$ and W. Khan ${ }^{1}$
}

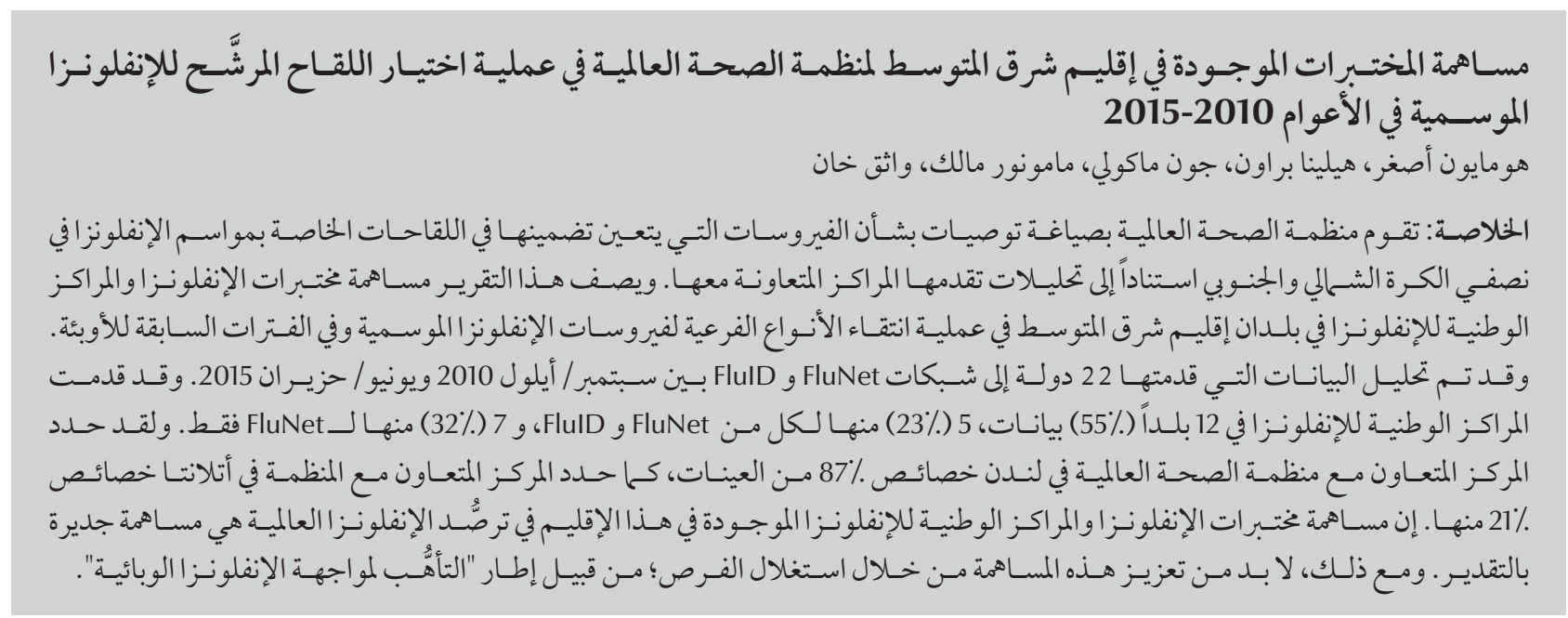

ABSTRACT The World Health Organization (WHO) formulates recommendations for viruses to be included in vaccines for the influenza seasons in the northern and southern hemispheres on the basis of analyses by its collaborating centres (CCs). This report describes the contribution of influenza laboratories and national influenza centres in countries in the WHO Region for the Eastern Mediterranean to the selection process of seasonal and pre-pandemic influenza virus subtypes. Data submitted by 22 countries to FluNet and FluID between September 2010 and June 2015 were analysed. National Influenza Centres (NICs) in 12 countries (55\%) reported data, 5 (23\%) to both FluNet and FluID and 7 (32\%) only to FluNet. The WHO CC in London characterized 78\% of the samples, and the CC in Atlanta, characterized 21\%. The contribution of influenza laboratories and NICs from this Region to global influenza surveillance is appreciable. However, enhancing the contribution through initiatives such as the Pandemic Influenza Preparedness Framework is still needed.

Contribution des laboratoires de la Région OMS de la Méditerranée orientale au processus de sélection du candidat vaccin contre la grippe saisonnière, 2010-2015

RÉSUMÉ L'Organisation mondiale de la Santé (OMS) émet des recommandations quant aux virus à inclure dans les vaccins contre les grippes saisonnières des hémisphères nord et sud, en fonction des analyses réalisées par ses centres collaborateurs. Le présent article décrit la contribution des laboratoires de la grippe et des centres nationaux de la grippe (CNG) des pays de la Région OMS de la Méditerranée orientale au processus de sélection des sous-types du virus de la grippe saisonnière et pré-pandémique. Les données transmises par 22 pays à FluNet et à FluID entre septembre 2010 et juin 2015 ont été analysées. Les CNG de 12 pays (55\%) ont transmis leurs données, dont $5(23 \%)$ à la fois à FluNet et à FluID, et 7 (32\%) à FluNet uniquement. Les centres collaborateurs de I'OMS de Londres et d'Atlanta ont caractérisé $78 \%$ et $21 \%$ des échantillons respectivement. La contribution des laboratoires de la grippe et des CNG de cette Région à la surveillance mondiale de la grippe est appréciable. Cependant, il est nécessaire de renforcer cette contribution en tirant parti d'opportunités telles que celle du Cadre de préparation en cas de grippe pandémique.

${ }^{7}$ WHO Regional Office for the Eastern Mediterranean, Cairo, Egypt (correspondence to Wasiq Khan: khanwa@who.int). ${ }^{2}$ Department of Pathology, University of Cambridge, Tennis Court Road, Cambridge, United Kingdom. ${ }^{3}$ Crick Worldwide Centre, The Francis Crick Institute, Mill Hill Laboratory, The Ridgeway Mill Hill, London, United Kingdom.

Received: 24/05/16; accepted: 23/08/16 


\section{Introduction}

Acute respiratory infections are a major cause of morbidity and mortality, with influenza virus infection a significant contributor, causing an estimated 250 000-500 000 deaths annually (1). Identification of representative candidate influenza viruses is an essential component of the global strategy for pandemic preparedness as the candidate viruses represent the basis for timely vaccine production for seasonal influenza, or in the event of a pandemic or potential pandemic influenza. Twice each year WHO formulates recommendations on the viruses to be included in influenza vaccines, one for the influenza season in the northern hemisphere and one for the southern hemisphere. The recommendations are reached through technical consultations in February and September each year. The process involves WHO collaborating centres (CCs) for influenza, WHO essential regulatory laboratories, WHO H5 reference laboratories and representatives of the animal sector (the World Organisation for Animal Health and the Food and Agriculture Organization of the United Nations). These partners consider epidemiological and epizoological data and the antigenic and genetic characteristics and antiviral susceptibility of recently circulating influenza viruses in order to recommend the most appropriate candidates for vaccines. Most of the results are derived from analyses carried out at the WHO CCs on viruses and samples provided by national influenza centres (NICs) around the world.

\section{Methods}

This report for the WHO Regional Office for the Eastern Mediterranean provides an assessment of the contribution made by the 22 countries in the Region to the selection process of seasonal and pre-pandemic influenza vaccines in
2010-2015. It describes the extent to which NICs and other laboratories in the Region provided influenza virus isolates, clinical specimens, gene sequence data and epidemiological information to the WHO CCs and submitted data to FluID (http://www.who.int/influenza/surveillance_monitoring/fluid/ en/) and FluNet (http://www.who. int/influenza/gisrs laboratory/flunet/ en/). The WHO CC laboratories conduct quantitative and qualitative analyses of human seasonal influenza viruses and clinical samples sent by countries to characterize specimens and screen for antiviral susceptibility. Sequences of haemagglutinin (HA) and neuraminidase (NA) and other genes from human influenza viruses are determined and submitted by the WHO CCs, NICs and other laboratories to the EpiFlu database platform provided by the global initiative on sharing of all influenza data (http://gisaid.org). Countries also provide zoonotic influenza samples to the WHO CCs for genetic, antigenic and other analyses for their potential use as pre-pandemic candidate influenza viruses for vaccines. Collaboration between influenza laboratories and NICs and the WHO CCs includes detailed testing of clinical samples and isolates, nucleotide sequencing of referred isolates, training and joint publications.

\section{Results}

\section{Virological surveillance and epidemiological reporting of seasonal influenza virus activity}

Twelve countries in the Region reported virological surveillance and epidemiological data. Bahrain, the Islamic Republic of Iran, Morocco, Oman and Pakistan submitted information to both FluNet and FluID; Afghanistan, Egypt, Iraq, Jordan, Qatar, the Syrian Arab Republic and Tunisia submitted data only to FluNet. Most countries submitted regular annual reports to FluNet during this period, although no reports were submitted to FluNet by the Syrian Arab Republic after 2012, and no data were received from Afghanistan or the Islamic Republic of Iran during the period 2010-2015.

Of the 22 countries in the Region, 15 have NICs that are recognized by WHO and during the period 20102015 shared seasonal influenza clinical samples and virus isolates with one of the WHO CCs (in London, Atlanta or Tokyo). During this period, a total of 1106 seasonal influenza virus samples were sent to the CCs by 12 countries. The majority (78\%) were sent to London, followed by $21 \%$ to Atlanta and $0.5 \%$ to Tokyo. Eleven of the 15 countries with recognized NICs sent influenza virus isolates or clinical samples, and the Global Influenza Surveillance and Response System (GISRS) Laboratory in Palestine (West Bank), which is not currently a recognized WHO NIC, sent $9 \%$ of all clinical samples; these were processed for virus isolation and characterization by the CC in London. Four of the countries with recognized NICs (Afghanistan, Kuwait, Lebanon and Somalia) did not send specimens to WHO CCs during the study period.

Most of the virus isolates (52\%) were sent from NICs in Egypt and Jordan (Figure 1); laboratories and NICs in Bahrain, the Islamic Republic of Iran, Morocco, Oman, Pakistan and Palestine provided 47-100 samples each (42\%), and Iraq, Qatar, the Syrian Arab Republic and Tunisia together contributed $6 \%$ of all the isolates received (6-30 samples).

The monthly and yearly contributions for the 12 countries that sent samples to the CCs are shown in Figure 2 (online). Although regular and consistent numbers of samples were sent during the 5-year period by all countries, notable increases and greater consistency were seen during the winter months. The NICs in Egypt, the Islamic Republic of Iran, Oman and Tunisia sent samples every year, whereas Bahrain, 
Jordan and Pakistan sent in 4 of the 5 northern hemisphere winter influenza seasons, Morocco in 3 seasons, Iraq in 2 seasons (2010-2011 and 2014-2015) and Qatar (2010-2011) and the Syrian Arab Republic (2011-2012) in 1 season each. The GISRS laboratory in Palestine provided samples during the winter of 2012-2013.

\section{Characterization of seasonal influenza virus samples by WHO collaborating centres}

Influenza virus isolates and clinical specimens provided by countries in the Region are characterized by the WHO CCs according to virus type, subtype and lineage, their ability to yield virus after culture in vitro and their sensitivity to licensed antiviral drugs. Influenza A viruses that cannot be assigned a subtype are designated "unsubtypeable", and influenza B viruses for which no lineage can be defined are designated "lineage not identified".

Of the virus types and subtypes received, $76.8 \%$ were type $A$, with $\mathrm{A}(\mathrm{H} 1 \mathrm{Nl})$ predominating over $\mathrm{A}(\mathrm{H} 3 \mathrm{~N} 2)$ viruses by a ratio of $1.3: 1$. Of the type B viruses (22.8\% of all specimens received), B-Yamagata lineage viruses predominated over those of the Victoria lineage by a ratio of $1.5: 1$. The proportion of samples to which no subtype (type A viruses) or lineage (type $B$ viruses) could be assigned varied considerably between countries, especially with regard to type A viruses (Figure 1); for example, $51 \%$ of type A samples from Egypt were designated "no subtype"; these samples were not classed as "unsubtypable", they usually had low virus load and the $\mathrm{H} 1$ and $\mathrm{H} 3$ primers and probes used to define subtypes were not sensitive enough for discrimination.

The seasonality of influenza in the Eastern Mediterranean Region is shown by the data on specimens received based on date of collection. Seasonality is also indicated by the higher frequency of shipments made during winter months.
Date of onset-based analysis of clinical cases and laboratory confirmed cases will be done separately in future.

Samples are received randomly and are fully characterized in advance of the WHO consultations on the influenza virus vaccine composition in February of each year for recommendations for the forthcoming northern hemisphere winter influenza season (2).

\section{Virus recovery rates and antiviral analysis of seasonal influenza virus samples}

The WHO CCs further characterize the influenza virus specimens received from laboratories in the Region by reinoculating virus isolates in cell cultures to obtain higher yields, i.e. samples that yield sufficient material for quantification by haemagglutinin and neuraminidase assay. Virus recovery was attempted for the majority (84\%) of samples received. Culture was not attempted from samples that contained virus levels deemed too low for sufficient recovery or samples that had been shipped in the same package with a suspected $\mathrm{A}(\mathrm{H} 5 \mathrm{N1})$ zoonotic virus. Virus was recovered from $75 \%$ of the samples that were cultured in vitro. The distribution of samples from countries from which virus was recovered is presented in Figure 3.

The WHO CC in London received only clinical samples from Palestine and Tunisia. The vast majority of specimens from the Islamic Republic of Iran (99\%) and all the samples from Qatar were provided as propagated viruses. Egypt, Iraq, Jordan, Morocco and Oman provided both clinical samples and propagated viruses, some as duplicates. Equivalent samples were not provided to the WHO CC in Atlanta.

Viruses that were recovered from tissue cultures were tested for sensitivity to the licensed neuraminidase inhibitors zanamivir and oseltamivir; $97 \%$ of the recovered isolates were sensitive (normal inhibition) to both drugs. The remaining 3\%, from the Islamic Republic of Iran, Jordan, Egypt, Morocco, Palestine and Qatar, could not be assayed for antiviraldrugsensitivitybecause ofinsufficient neuraminidase activity.

\section{Haemagglutinin gene sequences of seasonal influenza viruses}

Information on gene sequences, particularly for the HA gene, is used in conjunction with information on the antigenic properties of human influenza viruses in making the WHO recommendations for the composition of influenza vaccines (2).

The overall contribution of sequence information on influenza isolates from the Region was evaluated by searching the EpiFlu database for HA gene sequences from these countries. The sequences uploaded to the EpiFlu resource platform are summarized in Figure 4. The search yielded 563 fulllength HA gene sequences and 220 partial sequences (Table 1). Just over half $(51 \%)$ of the sequences were submitted by CCs and the other half by either NICs or academic research laboratories in 15 countries in the Region (Bahrain, Egypt, the Islamic Republic of Iran, Iraq, Jordan, Lebanon, Morocco, Oman, Pakistan, Palestine, Qatar, Saudi Arabia, Sudan, the Syrian Arab Republic and Tunisia). Of the total number of gene sequences (full-length and partial), 587 (75\%) were obtained from type A viruses and 196 (25\%) from type B viruses. H1 subtype sequences predominated over $\mathrm{H} 3$ sequences by a ratio of $1.6: 1$, and B/Victoria lineage virus sequences predominated over $\mathrm{B} /$ Yamagata sequences by a ratio of 2.3:1.

Although all information on virus genome sequences is useful, full-length sequences of the HA gene are generally considered more reliable for the phylogenetic analyses required for selection of the seasonal influenza virus vaccine strain; however, some of the tools used do not allow phylogenetic analysis of a mix of full-length and partial gene sequences. Most countries in the Region provided almost 100\% 
full-length sequences, except for the Islamic Republic of Iran, Lebanon, Morocco, Sudan, the Syrian Arab Republic and Tunisia. The vast majority of partial gene sequences submitted to EpiFlu were from laboratories that were neither WHO CCs nor NICs (Table 1).

\section{Zoonotic influenza viruses}

A further essential activity coordinated by WHO is the selection and development of candidate vaccine viruses from zoonotic sources that can cause a significant disease burden in humans, and the antigenic and genetic characterization of such viruses is an important step in pandemic preparedness (2). Between September 2010 and June 2015, influenza A(H5N1) viruses and clinical samples collected in Egypt were sent by the NIC to WHO CCs (43 to Atlanta, 4 to London and 2 to the WHO CC in Memphis, Tennessee, USA). Sequences of the HA genes from 30 influenza $\mathrm{A}(\mathrm{H} 5 \mathrm{~N} 1)$ viruses were submitted to the WHO EpiFlu database during this period.

There are currently 3 candidate $\mathrm{A}(\mathrm{H} 5 \mathrm{~N} 1)$ influenza vaccine viruses derived from samples collected in Egypt: A/Egypt/2321-NAMRU3/2007, A/Egypt/N03072/2010 and A/ Egypt/3300-NAMRU3/2008. All 3 were developed at the WHO CC in Atlanta and are available from that site (http://www.who.int/influenza/vaccines/virus/candidates_reagents/a_ h5n1/en/). Kayali et al. (3) give a detailed discussion of the contribution of research in laboratories in the Region to the epidemiology and molecular characterization of zoonotic influenza viruses.

\section{Training}

An important component of the collaboration between WHO CCs, NICs and other GISRS laboratories in the WHO Region for the Eastern Mediterranean is the opportunity for scientists from the Region to receive training at CC laboratories in specialized techniques
Table 1 Numbers of HA gene sequences submitted to EpiFlu by countries in the WHO Eastern Mediterranean Region, 2010-2015

\begin{tabular}{llr} 
Submitting laboratory & \multicolumn{1}{c}{ Country } & Full-length sequences \\
WHO Collaborating Centre, London & & 105 \\
& Jordan & 6 \\
& Egypt & 48 \\
& Islamic Republic of Iran & 39 \\
& Oman & 27 \\
& Palestine & 18 \\
& Iraq & 16 \\
& Morocco & \\
& Tunisia & \\
& Qatar & 330
\end{tabular}

WHO Collaborating Centre, Atlanta, Georgia

$\begin{array}{lr}\text { Bahrain } & 28 \\ \text { Egypt } & 13 \\ \text { Oman } & 15 \\ \text { Pakistan } & 10\end{array}$

Subtotal $\quad 66$

Islamic Republic of Iran, NIC $\quad 89$

Oman, academic/Austria $\quad 22$

Morocco, NIC 17

Lebanon, academic/Japan

Tunisia, NIC

Saudi Arabia, Ministry of Health

Jordan, academic/USA 2

Egypt, Ministry of Health

Saudi Arabia/Institut Pasteur, France $\quad 1$

Total 563

Partial sequences

Morocco, academic 83

Tunisia, NIC $\quad 50$

Lebanon, academic/Japan $\quad 24$

Islamic Republic of Iran, academic $\quad 23$

Sudan, Ministry of Science $\quad 20$

WHO Collaborating Centre, Tokyo

Syrian Arab Republic $\quad 6$

Morocco, NIC

5

WHO Collaborating Centre, London

Egypt 1

Qatar 1

Tunisia 1

Islamic Republic of Iran, NIC

Oman, academic/Austria 2

Palestine, academic

Saudi Arabia, academic, Ministry of

Health

Total 220

Overall total $\quad 783$

NIC = National Influenza Centre. 


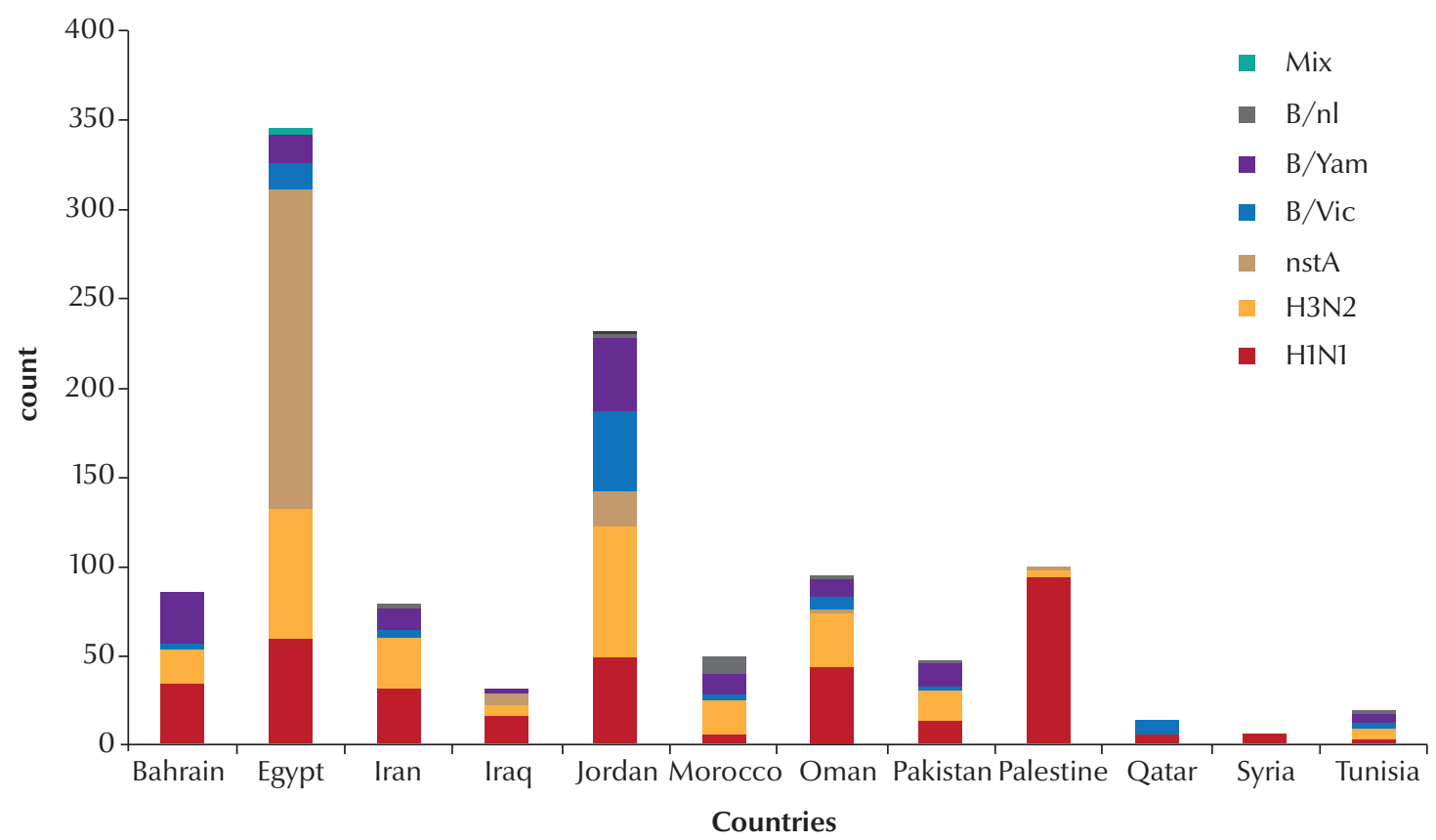

Figure 1 Number of seasonal influenza samples received from countries in the WHO Eastern Mediterranean Region by subtype and lineage, September 2010-June 2015

used in influenza surveillance, characterization, vaccine strain selection and research. In-house training modules are available on both virological and molecular biological techniques, including virus isolation; antigenic and serological analysis; assessment of sensitivity to anti-influenza drug compounds; and sequence analysis of influenza virus genomes.

Since 2010, a wide range of in-house advanced training in molecular techniques (e.g. polymerase chain reaction testing, sequencing and sequence analysis) or virological analysis (e.g. virus isolation and propagation, antigenic and serological analysis by haemagglutination inhibition, and antiviral sensitivity testing) has been delivered by the WHO CC in London to personnel of NICs in Iraq, Jordan, Morocco and Oman in a 2-week training course.

The CC in Atlanta delivered 2 training courses in Atlanta, with 2 participants from the Region; 1 course in Egypt, with 13 countries represented; 1 course in Thailand, with 1 participant from the Region; and 2 courses in Africa, with 3 participants from the
Region, 1 of which included participation by the London CC.

No training courses could be organised for the regional participants in the WHO CCs in Beijing, Melbourne, Memphis and Tokyo between 2010 and 2015 .

\section{Joint publications between WHO CCs and laboratories in the Eastern Mediterranean Region, 2010-2015}

The 24 joint publications during 2010-2015 are an indication of the extent of collaborative research between scientists in countries in the WHO Region for the Eastern Mediterranean and WHO CC laboratories. Most were with the $\mathrm{CC}$ for Studies on the Ecology of Influenza in Animals in Memphis and the Centers for Disease Control and Prevention in Atlanta. The majority of publications from the Eastern Mediterranean Region are from Egypt on influenza virus A(H5N1). The collaborative research needs to be expanded to cover other human and animal influenza viruses.

\section{Discussion}

Vaccines play an important role in global efforts for prevention and control and pandemic preparedness against influenza. To address influenza mortality and morbidity burden in communities, vaccination is currently the only practical means (4). The production of an optimal vaccine depends on monitoring of circulating and emerging influenza viruses at the global level. Influenza laboratories, particularly NICs, play a crucial role in this surveillance as part of the WHO GISRS. This report illustrates the continued contribution of NICs and influenza laboratories from the Region to the GISRS and to the selection process for seasonal influenza vaccine strains. Between September 2010 and June 2015, most countries in the Region contributed consistent epidemiological and surveillance information, influenza virus samples isolates and HA sequence data, all of which are of value to the $\mathrm{WHO}$ in selecting seasonal influenza vaccine strains and in the development of candidate influenza vaccine viruses. However, 


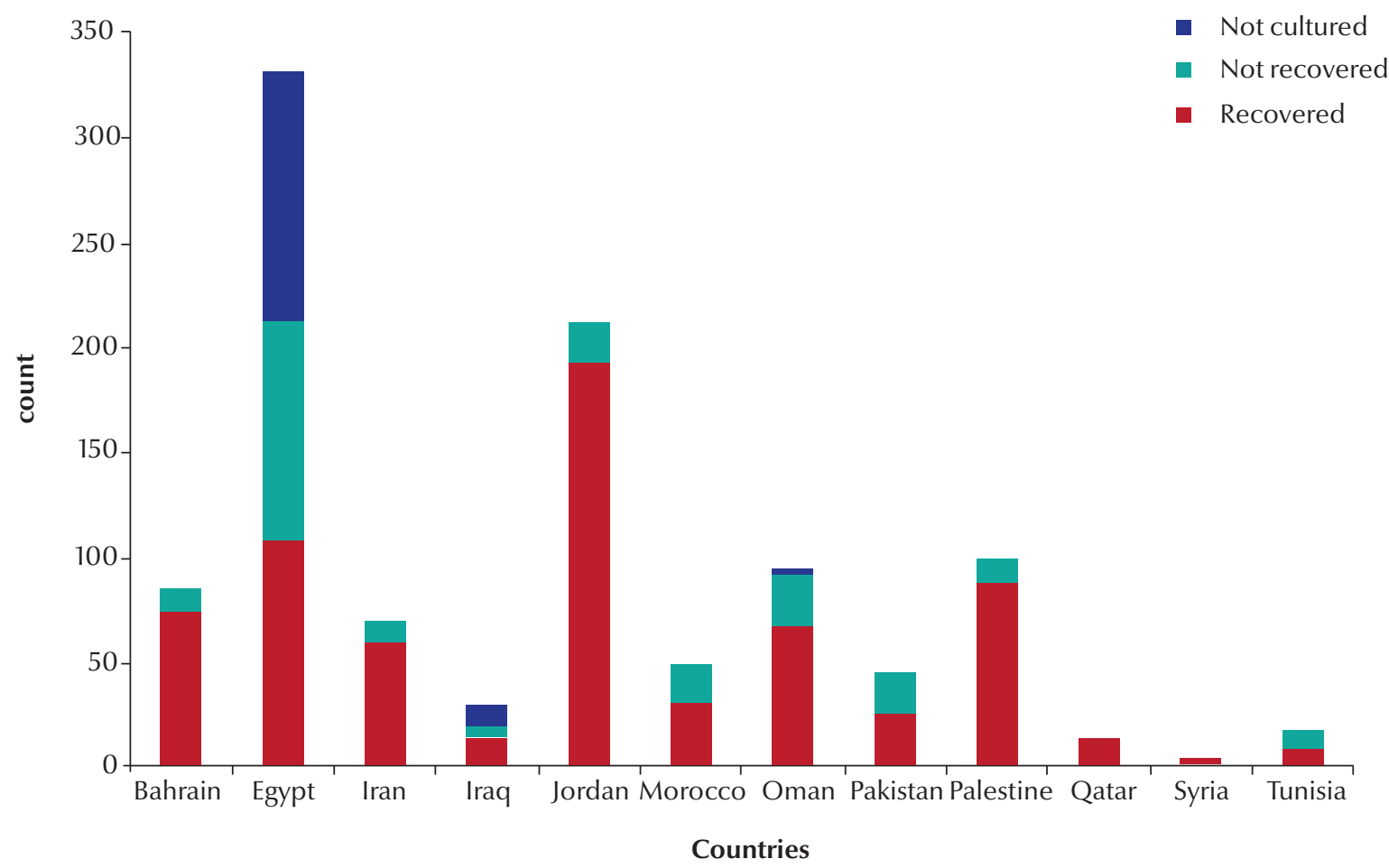

Figure 3 Distribution of of influenza virus isolates obtained after tissue culture inoculation for twelve countries of the WHO Region for the Eastern Mediterranean, September 2010-June 2015

NICs and influenza laboratories in this Region need to further enhance their contribution from all countries to the global process of vaccine strain selection and vaccine development. Research in the Region has shown some gaps; for example, collaboration between NICs and veterinary services to improve virological surveillance needs to be improved through concerted activities and follow-up (3).

The number of specimens sent from the Region to WHO CCs during the period we reviewed varied from country to country. The WHO CCs in London, Atlanta and Tokyo characterized significant numbers of virus samples from the Region and the CCs in London and Atlanta offered a range of advanced training modules or delivered courses to scientists from laboratories in the Region between 2010 and 2015. However, the circulation of avian influenza viruses in the Region and the emergence of Middle East respiratory syndrome coronavirus (MERS CoV) requires increased surveillance and enhanced virus sharing: this is an obligation for WHO Member States under the Pandemic Influenza Preparedness Framework, which calls for improving and strengthening the sharing of influenza viruses with human pandemic potential through GISRS (5).

This report shows there is consistent and productive collaboration between NICs, influenza laboratories and the WHO CCs. However, collaboration with the WHO CC in London appears to be more visible than other CCs because of its proximity to the Region, and over time collaboration in regard to sharing of specimens has been strengthened. Efforts should be coordinated by WHO to strengthen the relationship between regional NICs and influenza laboratories and other WHO CCs as a result of lessons learnt from the partnership with the London CC. This could reduce the burden on a single CC (London) and to serve as alternates, particularly in emergencies, as well as making it easier for countries to participate in training programmes organized by other WHO CCs.

Collaboration between scientists in the Eastern Mediterranean Region and WHO CCs is exemplified in the 24 joint papers published during the study period, although most of them are on avian influenza $\mathrm{A}(\mathrm{H} 5 \mathrm{~N} 1)$. These are listed at the end of this report. However, the research, particularly on virological surveillance, remains limited to certain countries (3). Efforts are needed to increase the amount of research conducted and published by the countries of the Region. The technical and financial support offered by WHO through the Partnership Contribution mechanism under the Pandemic Influenza Preparedness Framework is an opportunity to strengthen virological surveillance and research and support the NICs and influenza laboratories in the Region.

The WHO Region for the Eastern Mediterranean has become an "emergency” Region as more than 62 million 


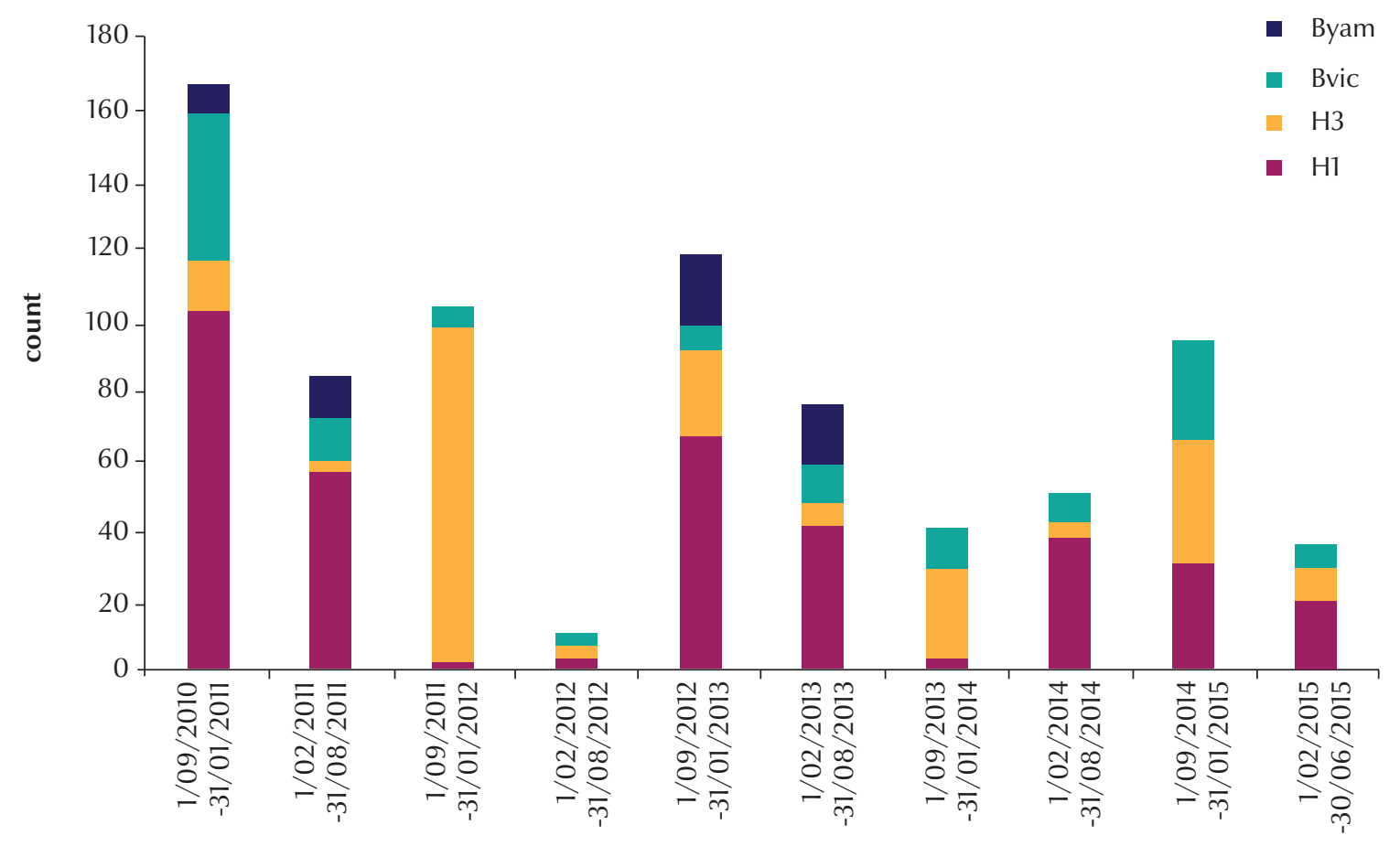

Collection period

Figure 4 Distribution of influenza subtypes for HA sequences submitted by countries of the WHO Region for the Eastern Mediterranean to EpiFlu, September 2010-June 2015 [Byam = B (Yamagata); Bvic = B (Victoria)]

people in this Region have been affected by natural disasters and emergencies arising from conflict (6). Despite the challenges faced by countries in the Region resulting from emergencies, the contribution of NICs and influenza laboratories to the selection process of candidate seasonal influenza vaccines is appreciable. However, a broader partnership of countries, WHO and technical partners such as the Centers for Disease Control and Prevention is needed to ensure continuity and to enhance existing virological surveillance and monitoring, regular and timely submission of isolates, and collaboration with WHO CCs and virological research laboratories in the Region.

\section{Acknowledgements}

We thank the directors and staff of the WHO CCs for providing the information for this report and the NICs and other laboratories who shared viruses and data with the CCs.

Funding: None.

Competing interests: None declared.

All figures available online at: http://www.emro.who.int/emh-journal/eastern-mediterranean-health-journal/home.html

\section{References}

1. Influenza (Fact sheet 211). Geneva: World Health Organization; 2014 (http;//www.who.int/mediacentre/factsheets/2003/ fs211/en/, accessed 31 August 2016).

2. A description of the process of seasonal and H5N1 influenza vaccine virus selection and development. Geneva: World Health Organization; 2007 (http://apps.who.int/gb/pip/ pdf_files/Fluvaccvirusselection.pdf?ua $=1$, accessed 31 August 2016).

3. Kayali G, Webby RJ, Samhouri D, Mafi AR, Bassili A. Influenza research in the Eastern Mediterranean Region: the current state and the way forward. Influenza Other Respir Viruses. 2013;7:914-21.
4. Barr IG, McCauley J, Cox N, Daniels R, Engelhardt OG, Fukuda $\mathrm{K}$ et al. Epidemiological, antigenic and genetic characteristics of seasonal influenza A (H1N1), A (H3N2) and B influenza viruses: basis for the $\mathrm{WHO}$ recommendation on the composition of influenza vaccines for use in the 2009-2010 Northern Hemisphere season. Vaccine. 2010;28(5):1156-67.

5. Fidler DP, Gostin LO. The WHO pandemic influenza preparedness framework: a milestone in global governance for health. JAMA, 2011;306(2):200-01.

6. Emergency preparedness and humanitarian action. Cairo: World Health Organization; 2016 (http://www.emro.who. int/eha/countries-in-crisis/index.html accessed 31 August 2016). 